\title{
Errorless learning and spaced retrieval techniques to relearn instrumental activities of daily living in mild Alzheimer's disease: A case report study
}

\author{
Stéphanie Thivierge ${ }^{1,2}$ \\ Martine Simard ${ }^{1,2}$ \\ Léonie Jean ${ }^{1,2}$ \\ Éric Grandmaison ${ }^{3}$ \\ 'School of Psychology, Laval \\ University, Quebec City, Quebec, \\ Canada; ${ }^{2}$ Centre de Recherche \\ Université Laval Robert-Giffard, \\ Quebec City, Quebec, Canada; \\ ${ }^{3}$ Neurosciences and Rehabilitation \\ Units, Moncton Hospital, Moncton, \\ NB, Canada
}

\begin{abstract}
Previous studies on cognitive training in Alzheimer's disease (AD) were principally aimed at making patients learn items not related to functional needs. However, AD patients also experience difficulties with instrumental activities of daily living (IADL). The goal of the present multiple baseline case report study was to assess the preliminary efficacy and tolerability of an individualized cognitive training program using the errorless learning (EL) and spaced-retrieval (SR) techniques to relearn forgotten IADLs in mild AD. Following an exhaustive neuropsychological assessment, two participants received two training sessions per week during four weeks. Participant A was trained to use his voice mail and Participant B, to manage the messages from his answering machine. The results showed that the program was well tolerated and improved performance on the trained tasks. These ameliorations were maintained over a 5 -week period. The effects of the training did not have any impact on global cognitive functions since the results on these measures remained relatively stable. This case report demonstrated preliminary efficacy of a new cognitive training program using EL and SR techniques tailored to the needs of AD patients. This is an important finding since the loss of these capacities alters autonomy in $\mathrm{AD}$ patients.
\end{abstract}

Keywords: Alzheimer's disease, errorless learning, spaced retrieval, memory, IADLs

\section{Introduction}

The prevalence of Alzheimer disease (AD) will increase considerably in the next decades in industrialized countries because of their aging populations. In the USA, there were 4.5 million Americans affected by AD in 2000, and it is estimated that this number will increase 3-fold by 2050 (Hebert et al 2003). In Canada, the prevalence estimates of the Canadian Study of Health and Aging suggest that 314,000 Canadians will present with AD in 2011 (Canadian Study of Health and Aging 1994). Current estimates indicate that 24 million individuals in the world present with dementia, and it is predicted that this number will double every 20 years (Qiu et al 2007).

At the moment, there is no cure for AD. However, several approaches are now available for the symptomatic treatment of AD. Cholinesterase inhibitors (ie, donepezil, rivastigmine, and galantamine) are effective to maintain cognitive abilities and to slow down cognitive and functional deteriorations in mild to moderate AD (Birks et al 2000; Birks and Harvey 2006; Loy and Schneider 2006; Simard and Sampson 2008). However, the pharmacological approach presents certain limitations (Evans et al 2004). Some patients do not respond to these compounds. It is difficult to know when to start, when to switch and when to stop treatment, and it may be hard to get the appropriate dose. Side effects, adverse events, and drug interactions may also occur. In addition, the costs are important for the patients and society (Evans et al 2004). For all these reasons, alternative treatment strategies were developed over the past fifteen years. 
Cognitive training is among these strategies. A recent meta-analysis looked at over 17 controlled studies on cognitive training in $\mathrm{AD}$, and concluded that this nonpharmacological approach is effective for improving cognition and function in AD (Sitzer et al 2006). In a systematic review of the literature, Grandmaison and Simard (2003) analyzed 17 studies on cognitive training in order to target techniques demonstrating some efficacy in AD. These authors concluded that the errorless learning (EL) technique, which supports the encoding of new material, and the spacedretrieval (SP) technique, which supports the recall of new learned material, were the most promising paradigms for training memory in AD. This conclusion was supported by another literature review (Bier et al 2006) that identified EL and SR as two out of three techniques to utilize for cognitive training in $\mathrm{AD}$, the third method being the vanishing cues. Briefly, the EL aims at reducing errors to a minimum during learning (Baddeley and Wilson 1994), whereas in the SR technique, the recall of the information is done by gradually increasing the delay between each correct recall (Camp et al 1996). The superiority of the EL technique over the errorful technique was recently demonstrated by Metzler-Baddeley and Snowden (2005), in four patients with AD, on tasks involving to relearn material that was previously familiar to the patients, and to learn new information. In another study, 25 patients with $\mathrm{AD}$ who received cognitive training registered statistically significant improvements compared to 19 control patients who received mental stimulation only (Loewenstein et al 2004). The gains were observed on the performance of cognitive tasks (ie, recall of facename associations, orientation, cognitive processing speed) and on the performance of functional tasks (ie, making change for a purchase) that were trained using the SR and other cognitive techniques such as dual cognitive support, procedural-memory activation, visuo-motor processing activation, and functional skills training. More recently, the EL and SR techniques used in combination to relearn forgotten names of celebrities and to learn new names showed some success with performances from $20 \%$ to $100 \%$ and from $0 \%$ to $100 \%$ in two patients with amnestic mild cognitive impairment (A-MCI) (Jean et al 2007). These results suggest that these two techniques used in combination are effective to learn and relearn episodic and semantic information in A-MCI patients. These patients are considered to be in an intermediate state between normal aging and early dementia (Petersen 2004).

Some authors criticized the fact that previous studies used multiple cognitive techniques to train the $\mathrm{AD}$ patients (often more than three), making it hard to determine the relative efficacy of each of them, and that only a few studies used performance-based measures of daily living functions or direct measures of the task to be trained (Grandmaison and Simard 2003; Sitzer et al 2006). The majority of previous studies on cognitive training in AD were principally aimed at making patients learn items not related to functional tasks. However, AD patients with mild-to-moderate dementia also experience difficulties with instrumental activities of daily living (IADL), such as difficulties with management of finances (Marson et al 2000), management of medication (Cotrell et al 2006), cooking skills (Baum and Edwards 1993), and communications skills such as using the telephone (Loewenstein et al 1995; Ala et al 2005). Although this has not been investigated as much as episodic memory training, some recent studies aimed to evaluate the impact of cognitive training on IADLs, tapping into procedural learning processes. Zanetti and colleagues (2001) obtained positive results (ie, a significant time reduction to carry out the task) in a controlled study involving the training of 13 basic and instrumental activities of daily living, and these results were essentially obtained by encouraging the participants to carry out the task, by modeling the task and by providing the patients with cues, reinforcement, as well as verbal and nonverbal prompts. However, this intervention was not tailored to the participants' needs. A case study, such as one conducted by Lekeu and colleagues (2002), may thus meet the patients' needs, by targeting a significant task for the participant. The intervention of Lekeu and colleagues (2002) involved the EL and SR techniques, and was successful in teaching two patients with mild AD to use their cell phone. According to the authors, this success could be attributed to spare procedural memory in the two patients. Despite a small sample size, this study introduces the possibility for patients with mild AD to effectively use EL and SR techniques to learn or relearn IADLs.

Impairments in IADLs are important in $\mathrm{AD}$, and the profiles of difficulties presented in early $\mathrm{AD}$ are heterogeneous (Bier et al 2006). Besides, the literature on cognitive training suggests that the benefits obtained following the intervention do not transfer to untrained tasks (Carney et al 1999; Cicerone et al 2000; Davis et al 2001). This underlines the importance to train relevant activities in each AD patient. In summary, some authors had already reported the efficacy of some learning methods in individualized cognitive training programs for AD. However these efficacy data were based only on a few studies (Adam et al 2000; Lekeu et al 2000; Clare et al 2001; Bier et al 2008). Therefore, the principal goal of the 
present study was to assess the preliminary efficacy of an individualized cognitive training program using the EL and SR techniques to relearn forgotten IADLs in mild AD. As a secondary goal, the present study was also designed to document the tolerability of the intervention in the patients and caregivers, since the burden of such an intervention has not yet been systematically assessed. The heterogeneity of AD patients' cognitive profiles, the variability of their functional needs and the goals of the present study support the choice of the case report as a relevant design for conducting the present research work. We thus performed a multiple-baseline across-subjects case report study.

\section{Ethical considerations}

The study was approved by the Ethics and Research Committee at Laval University ( $C E ́ R U L)$. Before entering into the study, patients and their caregivers were fully informed about the project and the risks of participating in it. They signed an informed consent that was approved by the CERUL. All nominative data were kept strictly confidential by coding of all documents.

\section{Case A}

Participant A was a 66-year-old man with 20 years of formal education. He was a priest, and he had taught philosophy in a college. At the time of the study, he had been retired for 7 years. He lived with a female friend who agreed to participate in the study as the principal caregiver. The patient and the caregiver were recruited at the Alzheimer Society (Quebec City section) during the summer of 2007 following a short presentation given by the first two authors of the present paper. Participant A had a familial history of $\mathrm{AD}$, as his father and brother had been both diagnosed with the disease. The medical records of Participant A revealed that he had AD, and did not present other neurological, psychiatric, vascular, or systemic disorder known to alter cerebral or cognitive integrity. At the time of the screening evaluation, Participant A did not have a current or previous history of alcohol or substance abuse. At the time of his involvement in this study, Participant A was taking donepezil (7.5 mg per day) since March 2007 and citalopram (10 mg per day) since the beginning of August 2007. He was thus stabilized on donepezil for more than three months when the study began, but was on citalopram $10 \mathrm{mg}$ per day since 16 days when he was assessed at baseline. When the cognitive training started, Participant A was still on donepezil (same dosage than baseline) and was also taking citalopram (10 mg per day) since 37 days. He remained on donepezil (7.5 mg per day) and citalopram (10 mg per day) up until the end of the study.

\section{Screening (diagnostic) evaluation and results}

A neuropsychological assessment was first conducted at the participant's home over two periods of 1 and a half hours, in order to assess cognitive functions and to determine which IADL could be trained. The diagnostic battery included the following tests administered to the patient: the Mini-Mental State Examination (MMSE) (Folstein et al 1975), Dementia Rating Scale-2 (DRS-2) (Mattis 2001), California Verbal Learning Test-2 (CVLT-2) (Delis et al 2000), Lexical and Semantic Fluency Tasks (Consortium of Montreal and McGill universities: Canadian Study of Health and Aging 1996), Boston Naming Test-30 item-version (BNT) (Kaplan et al 1983), Tower of London (ToL) (Culberston and Zillmer 2000), Trail Making Test (TMT) (Delis et al 2000), and Clock Drawing Test (CDT)-Command and Copy (Rouleau et al 1992; Tuokko et al 1992). The Neuropsychiatric Inventory (NPI) (Cummings et al 1994) and the Disability Assessment for Dementia (DAD) (Gélinas and Gauthier 1994) were completed with the caregiver per the administration manual of the scale. The DAD is a scale designed to assess functional ability in community residing individuals with dementia. Functional ability is measured through the assessment of basic, instrumental and leisure activities in various sections: hygiene, dressing, undressing, continence, eating, meal preparations, telephoning, going on an outing, finance, correspondence, medications, leisure and housework. In each section, intentions and actual actions are assessed separately. The scoring is dichotomic (Yes: 1 or No: 0) with the possibility of nonapplicable (N/A) responses. The total maximum score is 46 (with $0 \mathrm{~N} / \mathrm{A}$ ). The authors of the scale proposed a conversion of the scores into percentage (manual of the scale; Gélinas and Gauthier 1994). Good inter-rater $(\mathrm{ICC}=0.95)$ and test-retest reliability $(\mathrm{ICC}=0.96)$ as well as good internal validity ( $\alpha=0.96$ ) have been established (Gélinas and Auer 1996). This instrument allowed the selection of a problematic IADL in each participant.

The screening evaluation (see Table 1) revealed that Participant A was impaired in all the cognitive domains assessed. He had a MMSE score of 19, which is indicative of mild-to-moderate dementia per the normative data of Bravo and Hébert (1997). Relative to his age and education-matched cohort, Participant A's score on the DRS-2 fell in the severely impaired range. All the CVLT scores of Participant A were below -2.5 standard deviations, revealing severe amnesia. 
Table I Results of the cognitive and clinical assessments at screening

\begin{tabular}{|c|c|c|c|c|}
\hline \multirow[t]{2}{*}{ Cognitive domains/Tests } & \multicolumn{2}{|c|}{ Participant A } & \multicolumn{2}{|c|}{ Participant B } \\
\hline & Raw score & AEMSS* score & Raw score & AEMSS score \\
\hline \multicolumn{5}{|l|}{ Global cognitive functioning } \\
\hline \multicolumn{5}{|l|}{ DRS-2 } \\
\hline \multirow[t]{2}{*}{ Total score (max. I44) } & 108 & 0 & 119 & I \\
\hline & Raw scores & Z scores & Raw scores & $Z$ scores \\
\hline MMSE & 19 & $<-3.0$ & 25 & -1.79 \\
\hline \multicolumn{5}{|l|}{ Total score (max. 30$)$} \\
\hline \multicolumn{5}{|l|}{ Episodic memory } \\
\hline \multicolumn{5}{|l|}{ CVLT (French version) } \\
\hline List $A-$ Sum of trials I to 5 & 17 & -2.50 & 26 & -1.6 \\
\hline List B - Immediate recall & 0 & $<-3.0$ & 2 & -2.0 \\
\hline List A - Short term free recall & 0 & -2.5 & 0 & -2.5 \\
\hline List A - Short term cued recall & 3 & -3.0 & 2 & $<-3.0$ \\
\hline List A - Delayed free recall & 0 & -3.0 & 0 & -3.0 \\
\hline List A - Delayed cued recall & 0 & $<-3.0$ & 2 & -3.0 \\
\hline Recognition - True positives & 8 & $<-3.0$ & 15 & 0.5 \\
\hline Recognition - False positives & 17 & -3.0 & 19 & $<-3.0$ \\
\hline \multicolumn{5}{|l|}{ Semantic memory/Language } \\
\hline BNT (30-item version) & 21 & -2.0 & 15 & -2.0 \\
\hline \multicolumn{5}{|l|}{ Verbal fluency } \\
\hline Phonemic & 17 & -2.52 & 27 & -1.59 \\
\hline Semantic & 9 & -2.02 & 8 & -2.02 \\
\hline \multicolumn{5}{|l|}{ Executive functions } \\
\hline \multicolumn{5}{|l|}{ Tower of London } \\
\hline Total move score & 145 & $<-2.67$ & 27 & 0.8 \\
\hline \multicolumn{5}{|l|}{ Trail Making Test } \\
\hline Visual scanning & 73 & -3.0 & 36 & -1.0 \\
\hline Number sequencing & 150 & -3.0 & 27 & 1.33 \\
\hline Letter sequencing & 150 & -3.0 & 21 & 1.33 \\
\hline Number-Letter Switching & 240 & -3.0 & 91 & 0.67 \\
\hline Motor speed & 118 & -3.0 & 20 & 1.0 \\
\hline \multicolumn{5}{|l|}{ Clock Drawing Test } \\
\hline Copy (max. 10) & 5 & $<-3.0$ & 9 & 0 \\
\hline Free drawing (max. 10) & 6 & -2.63 & 10 & 0.95 \\
\hline \multicolumn{5}{|l|}{ Activities of daily living } \\
\hline DAD (\%) & $73 \%$ & & $86 \%$ & \\
\hline Neuropsychiatric symptoms & 18 & & 3 & \\
\hline NPI (max. I44) & & & & \\
\hline
\end{tabular}

Note: *Age and Education Corrected Scaled Score.

Abbreviations:

Participant A also presented significant impairments in semantic memory and language (as measured by the fluency tasks and the BNT), in executive functions (as measured by the ToL, TMT - Number-Letter switching condition and the Clock drawing [Command condition]), in visual tracking (as measured by the first three conditions of the TMT), and in visuo-construction (as measured by the Clock Drawing Test [Copy and Command conditions]). The NPI revealed the presence of dysphoria, anxiety, apathy, euphoria, and aberrant motor behavior. Participant A had difficulties with finances, 
correspondence, telephoning and going on an outing, and also with hygiene and dressing, as assessed by the DAD. Participant A thus presented cognitive, affective and behavioral alterations typically reported in $\mathrm{AD}$, and he indeed met the National Institute of Neurological and Communicative Disorders and Stroke and the Alzheimer's Disease and Related Disorders Association (NINCDS-ADRDA) criteria for probable AD (McKhann et al 1984). Considering the difficulties presented by the patient and the needs regarding IADLs expressed by his caregiver, we selected the utilization of the voice mail as the task to be trained. The caregiver informed the research team that the participant panicked every time he tried to use his voice mail. The caregiver and the patient wanted the utilization of the voice mail to be retrained mainly for security reasons.

\section{Evaluation of efficacy and tolerability}

The performance on the task to be trained was assessed by a direct measure of training (DMT). In order to adapt the training program to individual differences and needs, the form of the DMT was different in each participant. The DMTs were created from existing functional scales, chosen for their good psychometric qualities (Moore et al 2007) and their relevance to the task to be trained. The DMT of Participant A was adapted from the ADL Situational Test (Skurla et al 1988). This instrument contains four tasks (see Table 2). Each task has several items, and every item is scored according to the degree of assistance needed: $4=$ completes the task independently; 3 = requires verbal prompting; 2 = requires verbal and visual prompting; 1 = requires verbal, visual and physical prompting; and $0=$ does not complete the task. In the study of Skurla (1988), the ADL Situational Test was correlated with a measure of global cognitive functioning especially designed for the evaluation of $\mathrm{AD}$, the Clinical Dementia Rating Scale (CDRS) (Hugues et al 1982). The telephoning situation task was adapted to create the DMT of Participant A. Some of the 11 original items of the task were integrally kept (eg, "Picks up the receiver before dialing"), some were adapted (eg, "Attempts to use phone book" became "Look at the information card to find the number to dial"), and some items were added (eg, "Listen to the options"). The DMT of Participant A thus contained 16 items. The administration and scoring procedures were similar to those used in the original ADL Situational Test. The total score was reported in percentage (maximum possible raw score $=64$ ).

The general cognitive functioning was assessed by the DRS-2. This scale is valid and reliable to detect AD (Jurica et al 2001), is sensitive to cognitive deterioration associated with dementia over time (Salmon et al 1990), and indicates severity of dementia, (Shay et al 1991; Monsch et al 1995; Stuss et al 1996). The everyday memory functioning was assessed by the Rivermead Behavioral Memory Test (RBMT). The RBMT was developed to monitor change following treatment for memory disorders (Lezak 2004). The RBMTfour alternative forms permit to follow the evolution of the performance while controlling for practice effects. Three ways

Table 2 Description of the baseline instruments

\begin{tabular}{|c|c|c|c|}
\hline Instruments/Domain assessed & Sub-scales & Validity data & Reliability data \\
\hline $\begin{array}{l}\text { ADL Situational Test (Skurla et al } \\
\text { 1988)/Functional living skills }\end{array}$ & $\begin{array}{l}\text { Dressing for a cold and rainy day; } \\
\text { Making a cup of coffee; Purchasing } \\
\text { a snack and gloves; Telephoning } \\
\text { the pharmacy }\end{array}$ & $\begin{array}{l}\text { Total score was correlated with } \\
\text { CDRS }(r=0.60, p<0.05)\end{array}$ & None provided \\
\hline $\begin{array}{l}\text { DAFS (Loewenstein et al 1989)/ } \\
\text { Functional living skills }\end{array}$ & $\begin{array}{l}\text { Time orientation; communication } \\
\text { abilities; transportation; financial } \\
\text { skills; shopping skills; eating skills; } \\
\text { dressing/grooming skills. }\end{array}$ & $\begin{array}{l}\text { Performance of AD patients } \\
\text { correlated with the BDRS } \\
(r=-0.59, \mathrm{p}<0.0 \mathrm{I})\end{array}$ & $\begin{array}{l}\text { Inter-rater reliability of } 0.93 \text {; } \\
\text { Test-retest reliability from } 0.72 \\
\text { to } 0.91\end{array}$ \\
\hline DRS-II/General cognitive functioning & $\begin{array}{l}\text { Attention; Initiation/Perseveration; } \\
\text { Construction; Concepts; Memory }\end{array}$ & $\begin{array}{l}\text { Score correlated with MMSE } \\
(r=0.82)\end{array}$ & $\begin{array}{l}\text { Test-retest reliability of } 0.97 \text { for } \\
\text { the Total Score }\end{array}$ \\
\hline RBMT/Everyday memory functioning & $\begin{array}{l}\text { First and Second Name; Belonging; } \\
\text { Appointment; Pictures; Story; } \\
\text { Faces; Route; Message; Orientation } \\
\text { and Date }\end{array}$ & $\begin{array}{l}\text { Score correlated with observed } \\
\text { memory lapses ( } r=-0.7 \text { I for } \\
\text { the Screening Score and }-0.75 \\
\text { for the standardized Profile } \\
\text { Score, } p<0.00 \text { I) }\end{array}$ & $\begin{array}{l}\text { Inter-rater reliability of } 1.00 ; \\
\text { parallel-form reliability from } 0.83 \\
\text { to } 0.88 \text { for the standardized Profile } \\
\text { Score; Test-retest reliability of } 0.85 \\
\text { for the Profile Score }\end{array}$ \\
\hline DQoL/Quality of life & $\begin{array}{l}\text { Self-esteem; Positive affect/ } \\
\text { humour; Negative affect; Feelings } \\
\text { of belonging; Sense of aesthetics }\end{array}$ & $\begin{array}{l}\text { The negative affect scale } \\
\text { correlated with the Geriatric } \\
\text { Depression Scale }(r=0.64)\end{array}$ & $\begin{array}{l}\text { Internal consistency ranging from } \\
0.67 \text { to } 0.89 ; \text { Test-retest reliability } \\
\text { ranging from } 0.64 \text { to } 0.90\end{array}$ \\
\hline
\end{tabular}

Abbreviations: 
of scoring are possible: the raw score, the standardized profile score and the screening score. This instrument has good validity, perfect inter-rater reliability, good parallel-form reliability and good test-retest reliability (Wilson et al 1989). Finally, the Dementia Quality of Life instrument (DQoL) is a selfreported measure on the quality of life designed for patients with dementia presenting an MMSE $>12$. This instrument has moderate to high internal consistency between the different scales and good convergent validity and test-retest reliability (Brod et al 1999; Schölzel-Dorenbos et al 2007).

The patient's tolerability was assessed using the attendance at the different training sessions, the attendance and regularity of the practices (as assessed by the practice journal) and the feedback provided by the participant and his caregiver (also documented in the practice journal). The caregiver's tolerability was assessed using his feedback, the observation of his ability to practice the task with the participant, and by his attendance at the patient's practice sessions (as assessed by the practice journal).

\section{Establishment of the baseline level of performance and general procedure}

After the screening evaluation, a baseline evaluation was carried out using the DMT, at three different moments, over a 2-week period, to establish a reliable baseline level of performance before the introduction of the cognitive training. The number of three baseline assessments was chosen for best reliability as a minimum of two baseline assessments is usually required (Levine and Downey-Lamb 2005). The other baseline evaluations were realized in order to assess the general cognitive functioning (DRS-2), everyday memory functioning (RBMT), and quality of life (DQoL). Then, the chosen task was trained during two sessions per week for four weeks, during which EL and SR techniques were applied. The DMT was thus administered at baseline, at the end of each training sessions, in order to follow the progression of learning, and at the two follow-ups. The other instruments of the baseline evaluation (RBMT-alternate forms, DQoL) were re-administered at the end of the training and at the two follow-ups performed one (FU 1) and five (FU 2) weeks after completion of the training program. Because it has only one alternative form, the DRS-2 was re-administered only at the first follow-up.

\section{Cognitive training}

The intervention was implemented at week 2 following the third baseline assessment on the DMT (see Figure 1). Participant A was trained to use his voice mail. The intervention consisted of two sessions per week, each lasting from

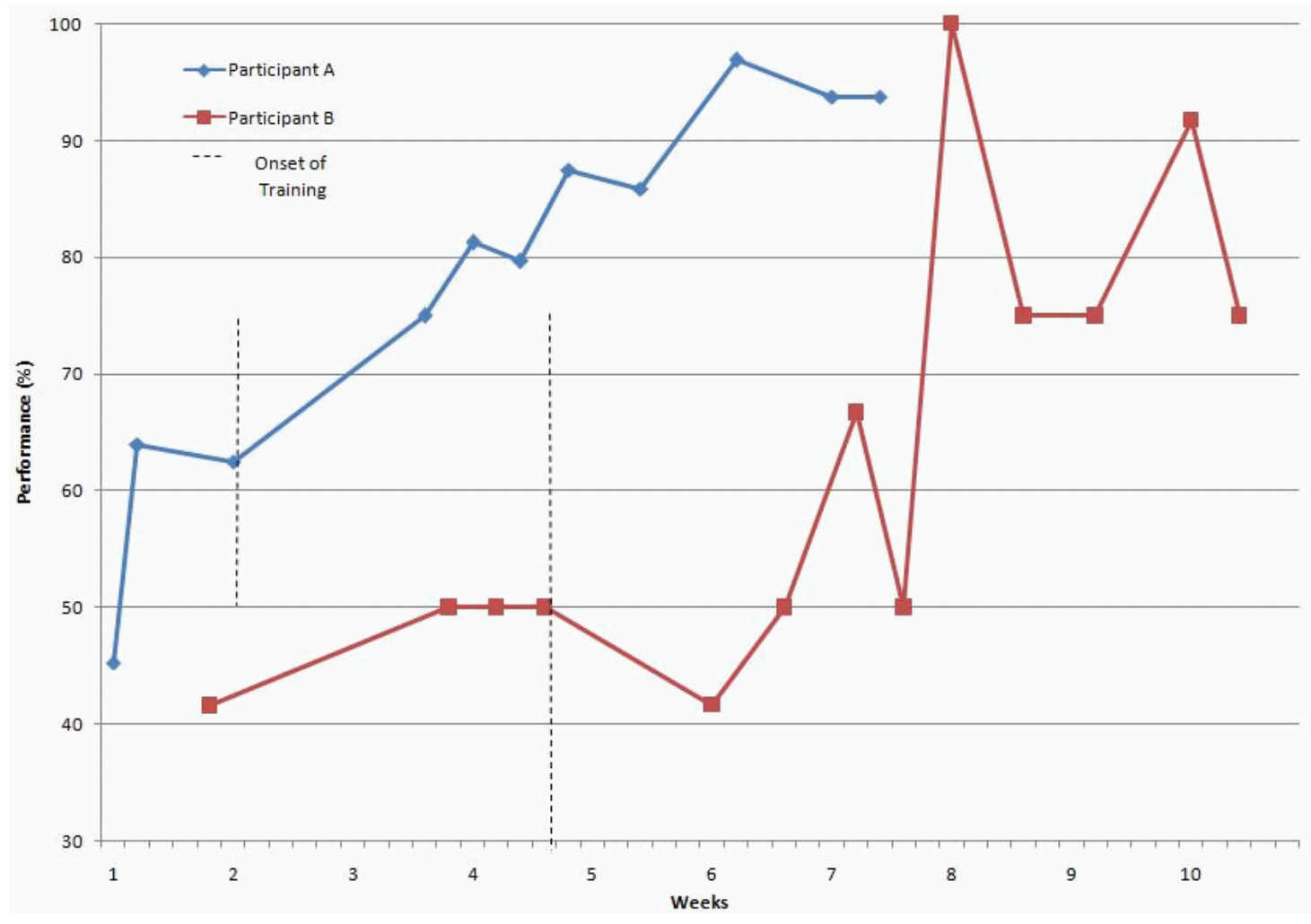

Figure I Performance of participants on the direct measure of training at baseline and at each training session. 
45 minutes to 1 hour. This training was delivered by a PhD candidate (ST) supervised by a neuropsychologist (MS). In order to ensure that the participant did not commit errors during the learning (errorless learning paradigm), decreasing degrees of assistance were provided, adapted to the patient's performance. The four levels of assistance were: 1) the assistant carried out the task in front of the participant; 2) the assistant named each step of the task to be carried out and the participant executed each of these steps; 3 ) the participant named all the steps of the task and carried out these steps with the help of the assistant, if needed; 4) the participant carried out the task independently. In order to facilitate the retrieval of information, expanded delays (30 seconds, 1 minute, 2 minutes, 4 minutes and 8 minutes) were inserted between each correct realization of the task (spaced retrieval paradigm). When an incorrect performance occurred, a return to the previous time interval was made, and the previous level of assistance was restored (for example: if at the 4-minute interval, assistance 3 was given and an error occurred, the next trial was at an interval of 2 minutes and assistance 2 was given). At the next successful trial, the interval time was increased once again, but only by half of the next time interval initially planned. The participant was asked to practice the task at least three times a week outside of the cognitive training program, under the supervision of his caregiver. The caregiver was trained to use the cognitive training techniques by the $\mathrm{PhD}$ candidate and a manual including information on memory systems, $\mathrm{AD}$, learning techniques and their application was also provided. The caregiver was asked to fill out a practice journal, in order to provide information and assistance as needed, to verify the tolerability of the training program, and also to ensure that the practices took place each week of the program. The caregiver of Participant A mentioned that she continued to practice with the patient, one to two times per week, during 3 weeks, after the end of the intervention phase.

\section{Results of training}

Figure 1 illustrates the scores obtained on the DMT by Participant A at each baseline measurement and at every training session. The vertical line on Figure 1 represents the onset of training. Table 3 presents the raw data and percentages obtained on the DMT by Participant A at each baseline measurement and at every training session as well as at Follow-ups 1 and 2. Table 4 indicates the results of the Participant A at baseline, post-intervention and at follow-ups on the DRS-2, the RBMT, and the DQoL.

Participant A performed with a mean of $57.3 \%$ on the baseline measures. The training was introduced when his performance was in decline (see Figure 1 and Table 3). His performance improved from $62.5 \%$ to $75.0 \%$ with the introduction of the intervention, and showed a constant amelioration during the training program. Participant A completed the training sessions with a performance of $93.7 \%$. His best performance was of $96.9 \%$ at training session \#6. He was able to maintain his new skill over a period of five weeks, since his performances at Follow-ups 1 and 2 reached, respectively, $90.6 \%$ and $89.1 \%$ on the DMT (the FU results are not shown on Figure 1). In order to strengthen this visual analysis, we calculated the effect size of the intervention using the Common Language Effect-Size (CLES) statistic (McGraw and Wong 1992). This statistic is comparable to Cohen's $d$, and generates a $\mathrm{Z}$ score for the difference between the two means in case reports. This $Z$ score is then converted to a probability from a $Z$ table: $Z_{C L}=\left(M_{1}-M_{2}\right)$ / $\sqrt{ }\left(\operatorname{Var}_{1}+\operatorname{Var}_{2}\right)($ Parker and Hagan-Burke 2007). We contrasted the baseline score $\left(\mathrm{M}_{1}\right)$ with the scores of the intervention and follow-up phases $\left(\mathrm{M}_{2}\right)$. Participant A obtained the following results: $\mathrm{M}_{1}=57.30, \mathrm{M}_{2}=88.42, \operatorname{Var}_{1}=108.64, \operatorname{Var}_{2}=32.97$ and $Z_{C L}=-31.12 / 11.19=-2.61$, which is a 0.9955 probability in a normal $Z$ distribution. Therefore, a $99.55 \%$ chance exists that any treatment or follow-up scores will be higher than any baseline scores. His scores on the DRS-2 and on the DQoL remained relatively stable. However, scores on the RBMT fluctuated through the evaluations (see Table 4).

The training program was well tolerated by the participant and his caregiver since they did not miss any evaluations, training sessions or follow-ups. Participant A did not express signs of fatigue or overwhelming anxiety or distress in any

Table 3 Results of participants A and B on the direct measure of training at each training session

\begin{tabular}{lll}
\hline Sessions & Participant A & Participant B \\
\hline Baseline I & $29 / 64(45.3 \%)$ & $5 / / 2(4 I .7 \%)$ \\
Baseline 2 & $4 \mathrm{I} / 64(64.1 \%)$ & $6 / 12(50.0 \%)$ \\
Baseline 3 & $40 / 64(62.5 \%)$ & $6 / 12(50.0 \%)$ \\
Baseline 4 & $\mathrm{n} / \mathrm{a}$ & $6 / 12(50.0 \%)$ \\
Training session I & $48 / 64(75.0 \%)$ & $5 / 12(41.7 \%)$ \\
Training session 2 & $52 / 64(81.3 \%)$ & $6 / 12(50.0 \%)$ \\
Training session 3 & $5 \mathrm{I} / 64(79.7 \%)$ & $8 / 12(66.7 \%)$ \\
Training session 4 & $56 / 64(87.5 \%)$ & $6 / 12(50.0 \%)$ \\
Training session 5 & $55 / 64(85.9 \%)$ & $12 / 12(100.0 \%)$ \\
Training session 6 & $62 / 64(96.9 \%)$ & $9 / 12(75.0 \%)$ \\
Training session 7 & $60 / 64(93.7 \%)$ & $9 / 12(75.0 \%)$ \\
Training session 8 & $60 / 64(93.7 \%)$ & $\mathrm{II} / 12(91.7 \%)$ \\
Training session 9 & $\mathrm{n} / \mathrm{a}$ & $9 / 12(75.0 \%)$ \\
Follow-up I & $58 / 64(90.6 \%)$ & $9 / 12(75.0 \%)$ \\
Follow-up 2 & $57 / 64(89.1 \%)$ & I0/I2 (83.3\%) \\
\hline
\end{tabular}


Table 4 Results of participants on the DRS-2, RBMT, and DQoL

\begin{tabular}{|c|c|c|c|c|}
\hline & \multicolumn{4}{|c|}{ Participant A } \\
\hline & Baseline & Post-intervention & Follow-up I & Follow-up 2 \\
\hline \multicolumn{5}{|l|}{ DRS-2 } \\
\hline Total score (max. 144) & 108 & & 107 & \\
\hline Attention (max. 37) & 33 & N/A & 34 & $\mathrm{~N} / \mathrm{A}$ \\
\hline Initiation/Perseveration(max.37) & 25 & & 21 & \\
\hline Construction(max. 6) & 6 & & 6 & \\
\hline Conceptualization (max. 39) & 33 & & 36 & \\
\hline Memory (max. 25) & 11 & & 10 & \\
\hline \multicolumn{5}{|l|}{ RBMT } \\
\hline Standardised profile score (max. 12) & 5 & 4 & 0 & 5 \\
\hline Screening score (max. 24) & 2 & I & 0 & I \\
\hline \multicolumn{5}{|l|}{ DQoL } \\
\hline Total score (max. 145) & 130 & 129 & 129 & 130 \\
\hline Self-esteem (max. 20) & 19 & 19 & 17 & 18 \\
\hline Positive affect/humour(max. 30) & 29 & 28 & 28 & 28 \\
\hline Negative affect (max. 55) & 46 & 49 & 51 & 49 \\
\hline Feelings of belonging (max. I5) & 13 & 14 & 13 & 14 \\
\hline \multirow[t]{2}{*}{ Sense of aesthetics $(\max .25)$} & 23 & 19 & 20 & 21 \\
\hline & \multicolumn{4}{|c|}{ Participant B } \\
\hline \multicolumn{5}{|l|}{ DRS-2 } \\
\hline Total score & 119 & & 123 & \\
\hline Attention & 36 & & 37 & \\
\hline Initiation/Perseveration & 29 & N/A & 32 & $\mathrm{~N} / \mathrm{A}$ \\
\hline Construction & 5 & & 6 & \\
\hline Conceptualization & 37 & & 32 & \\
\hline Memory & 14 & & 16 & \\
\hline \multicolumn{5}{|l|}{ RBMT } \\
\hline Standardized profile score & 6 & 3 & 9 & 5 \\
\hline Screening score & 0 & 0 & 3 & 3 \\
\hline \multicolumn{5}{|l|}{ DQoL } \\
\hline Total score & 112 & 106 & 104 & 110 \\
\hline Self-esteem & 17 & 17 & 16 & 20 \\
\hline Positive affect/humor & 24 & 23 & 24 & 26 \\
\hline Negative affect & 48 & 46 & 44 & 42 \\
\hline Feelings of belonging & 10 & 10 & 9 & 12 \\
\hline Sense of aesthetics & 13 & 10 & 11 & 10 \\
\hline
\end{tabular}

Abbreviations:

parts of the intervention. The caregiver did not report an increase of neuropsychiatric symptoms in Participant A. The caregiver also mentioned that she witnessed amelioration on the trained task as the cognitive intervention progressed. The caregiver well understood and correctly applied the principles of EL and SR following her training with the first author of the present paper. She was able to practice the task with the participant, as demonstrated by the practice journal and the feedback she provided to the first author (ST). She did not complain about the time she had to devote to the study, and did not report an increase of burden.

\section{Case B}

Participant B was a 68-year-old man with 17 years of formal education. He had been a civil engineer before his retirement. At the time of his involvement in the study, he had been retired 
for 14 years. He lived alone in the community, but his son agreed to get involved in the study as the principal caregiver. Participant B had a familial history of AD since his father had been diagnosed with the disease before dying. The medical records of Participant $B$ revealed that apart from $A D$, he did not present other neurological, psychiatric, vascular, or systemic disorder known to alter cerebral or cognitive integrity, and he did not have a current or previous history of alcohol or substance abuse. At the time of his involvement in this study, Participant B was taking galantamine $16 \mathrm{mg}$ per day since at least three months.

\section{Screening (diagnostic) evaluation and results}

Participant B underwent the same neuropsychological and clinical assessment as Participant A (see Table 1). He had a MMSE total score of 25, indicating mild dementia (per age and education adjusted score; Bravo and Hébert 1997). Relative to his age and education-matched cohort, his score on the DRS-2 fell in the severely impaired range. His performance on the CVLT revealed severe episodic memory problems. Participant $\mathrm{B}$ also presented impairments in semantic memory and language (as measured by the BNT and the fluency tasks). However, executive functions (as measured by the ToL, the TMT, and the CDT), were still in the normal range at the time of the screening evaluation. Participant B presented mild symptoms of euphoria and disinhibition on the NPI. The DAD revealed problems with the utilization of the telephone and the management of finances, correspondence, medication, and housework. Participant B thus met the NINCDS-ADRDA diagnostic criteria for probable AD (McKhann et al 1984). Taking into account the difficulties presented by the patient as expressed by his caregiver, we decided to select the management of the messages from his answering machine as the task to be trained. Prior to the training, Participant B was able to correctly note his messages, but he did not erase them on the answering machine, and kept writing notes about old and new messages. As a result, he was overwhelmed, confused and stressed by the high number of notes he had written, and by the presence of several new and old messages on his answering machine. He never remembered that he had already listened to a message and that he had already responded to this message, and could, for example, call back two or three times the same person in relation to a message left on his answering machine.

\section{Evaluation of efficacy and tolerability}

The performance on the task to be trained was assessed by a DMT. The DMT of Participant B was derived from the Direct
Assessment of Functional Status (DAFS) (Loewenstein et al 1989). This instrument assesses the functional performance in seven domains (see Table 2). For each individual item of a subscale, a score of 0 (incorrect performance) or 1 point (correct performance) is given. This instrument showed high interrater and test-retest reliability, and the performance of AD patients on the DAFS significantly correlated with the Blessed Dementia Rating Scale (BDRS) (Blessed et al 1968) in the study of Lowenstein and colleagues (1989). The DMT of Participant B was adapted especially from the 'Using the telephone' task of the DAFS to assess the capacity to manage the messages on the answering machine. Twelve items tapping into this domain were created. Participant B was thus evaluated on his ability to manage three types of message: 1) an appointment; 2) news; 3) or something he has to do in the future (such as calling somebody during the week). As with the DAFS, 0 or 1 point was granted for each item. In order to facilitate comparisons between Participants A and $\mathrm{B}$, the total score of the DMT of Participant B was reported in percentage (maximum possible raw score $=12$ ).

In addition, Participant B was administered, as was Participant A, with the DRS-2, the RBMT and the DQoL (see Table 2). The tolerability was assessed in a similar manner in Participants A and B.

\section{Establishment of the baseline level of performance and general procedure}

The baseline evaluation was carried out, after the screening evaluation, at four different moments, over a 4-week period, on the DMT (see Figure 1 and Table 3). The other baseline evaluations were realized in order to assess the general cognitive functioning, everyday memory functioning, and quality of life. Then, the chosen functional task was trained during two sessions per week for four weeks, during which EL and SR techniques were applied. The instruments of the baseline evaluation were re-administered at the end of the training and at the 2 follow-ups performed one (FU 1) and five (FU 2) weeks after completion of the training program.

\section{Cognitive training}

Participant B was trained to manage the messages from his answering machine. The cognitive training was implemented at different times for each participant because if the performance of the trained participant increased while the performance of the untrained participant remained at the baseline level, the amelioration could reasonably be imputed to the introduction of the cognitive training (Kazdin 1982; Barlow and Hersen 1984). Therefore the cognitive training of 
Participant B started at week 4, following the fourth baseline assessment on the DMT. Except for the DMT, the same cognitive training procedures as Participant A were implemented for Participant B. However, the caregiver did not continue to practice with Participant $B$ following the end of the intervention phase as the Participant A's caregiver did.

\section{Results of training}

Participant B had a mean performance of $47.9 \%$ at baseline. Although he was stable on the last three baseline measurements per the DMT (see Figure 1 and Table 3), his performances fluctuated throughout the training sessions. However, he reached a perfect score at training session \#5 (100.0\%). A ninth training sessions was added because of his fluctuating performances on the DMT. He completed the training phase with a score of $75.0 \%$ on the DMT. He also maintained his new skill, as demonstrated by the percentages he obtained at the follow-ups, (ie, $75.0 \%$ at Follow- up 1 and $83.3 \%$ at Follow-up 2 (the FU results are not shown on Figure 1). As for Participant A, we calculated the effect size of the intervention with the CLES statistic. Participant B obtained the following results: $M_{1}=47.92, M_{2}=73.26, \operatorname{Var}_{1}=17.22, \operatorname{Var}_{2}=227.23$ and $Z_{C L}=-25.34 / 15.63=-1.62$, which corresponds to a 0.9474 probability in a normal $\mathrm{Z}$ distribution. Therefore, a $94.74 \%$ chance exists that any treatment or follow-up scores will be higher than any baseline scores. The scores on the DRS-2 and the DQoL were relatively stable. However, the performances on the RBMT fluctuated (see Table 4).

Participant B well tolerated the training program. He did not miss any evaluations, training sessions or follow-ups. Participant B did not express signs of fatigue or overwhelming anxiety or distress during any parts of the intervention and his caregiver did not report an increase of neuropsychiatric symptoms. The caregiver also mentioned that he witnessed amelioration on the trained task as the cognitive training progressed. The caregiver was always present when it was necessary. The principles of the learning techniques were well understood and were also applied appropriately by the caregiver, and as a result, the caregiver regularly practiced the task to be trained with the participant, as demonstrated by the practice journal and the feedback he provided to the first author of the study (ST). Participant B's caregiver did not report an increase in burden, and he also did not mention that the study was too time-consuming.

\section{Discussion}

This case report study demonstrated that it is possible, for patients with $\mathrm{AD}$, to relearn forgotten IADLs using the EL and SR techniques. Both participants improved considerably on the DMT, and reached perfect or nearly perfect performances during training (Participant $\mathrm{A}=96.9 \%$; and Participant $\mathrm{B}=100 \%$ ). In addition, the results of the effect size calculations provided an objective measure of the intervention' strength (Parker and Hagan-Burke 2007). While the performance of Participant A improved with the introduction of the cognitive training, the performance of Participant $\mathrm{B}$ (still untrained at the time) remained relatively stable, indicating that the performance of Participant A was not improved or influenced by extraneous factors (eg, maturation, history or testing) (Backman et al 1997; Kazdin 2002). Moreover, the proposed intervention was well tolerated by both participants and their caregivers. To our knowledge, tolerability has not been formally measured in similar case studies.

Although Participant B was in a milder stage of AD, he did not ameliorate the skill to relearn right after the introduction of the cognitive training; instead his learning curve fluctuated, and he completed training with a score below that of Participant A. This can possibly be explained by the fact that the practices with the caregiver of Participant B started later and were less frequent than those of Participant $\mathrm{A}$, as revealed by the practice journal. Participant A started to practice with his caregiver in the first week of the training phase, and he practiced the skill three to four times per week with his caregiver, whereas Participant B began to practice with his caregiver in the second week of the training phase, and he only received two to three practices per week. The caregiver for Participant B was his son, and did not live with the patient as did Participant A's caregiver. Moreover, it is possible that the DMT of Participant A was more sensitive to the improvement since it allowed scoring to be graded from 0 to 4 points according to the assistance required by the participant for a correct realization of each part of the activity. The pass or fail mode of scoring on Participant B's DMT might not have reflected the range of change between a failed performance and a successful one.

The cognitive training did not increase general cognitive functioning, everyday memory functioning and quality of life as measured by, respectively, the DRS-2, the RBMT, and the DQoL. In both participants, there were some fluctuations in the results of the RBMT, but there was no fluctuation in the results of the memory subscale of the DRS-II. The fluctuating RBMT scores of Participants A and B did not indicate an overall amelioration or deterioration in everyday memory performance. It is interesting to note that these fluctuations were not found in a longitudinal analysis of memory functioning in patients with schizophrenia assessed with the 
RBMT at baseline, 9 months and 18 months later (Tyson et al 2005). This absence of variation in the RBMT scores in another clinical population suffering from a nondegenerative disorder, combined with the good parallel-form reliability and good test-retest reliability (Wilson et al 1989) established for the RBMT, strongly suggest that these fluctuations could perhaps be due to the characteristics of the AD population, to the cognitive training, or both. However, without a control group, it is difficult to determine whether the fluctuations were caused by the cognitive training or by natural progression of $\mathrm{AD}$ in which fluctuations are frequently reported (Bradshaw et al 2004).

Although this kind of cognitive training has not been largely investigated in previous studies, the present results are consistent with some findings reported in the literature. Based on the preservation of procedural memory in AD, Zanetti and colleagues (2001) and Lekeu and colleagues (2002) demonstrated that it is possible to have functional gains following the cognitive training of ADL in patients with AD. However, Zanetti and colleagues (2001) concluded that their intervention was effective on the basis of a decrease in the total completion time for the realization of the 13 ADLs trained. Of these 13 ADLs, there were basic (eg, dressing) and instrumental (eg, using the telephone) ADLs. Since the authors did not give the individual completion time registered for each ADL or the completion time registered for the basic ADLs and the instrumental ADLs, it is impossible to determine the contribution of each activity in the improvement obtained following the intervention. It is possible that the intervention had little effect on the instrumental ADLs. In addition, a decrease in the time required to complete a task doesn't mean that the patient improved his ability to correctly perform the activity. These are important findings but of little clinical utility. On the contrary, the present study demonstrated that AD patients can improve their ability to realize an IADL. The intervention program is one among the few that have been tailored to the difficulties presented by each patient, as expressed by his caregiver. This last particularity is of crucial importance for the clinical application of the intervention. The gains obtained by cognitive training are specific to the trained material because the improvement did not generalize to the cognitive functions measured.

In a recent study assessing the efficacy of a cognitive training program for $\mathrm{AD}$ patients receiving cholinesterase inhibitors, Loewenstein and colleagues (2004) argued that cognitive training programs should focus specifically on the training of functional tasks, rather than simply targeting general theoretical cognitive constructs. These authors found that their participants did not improve their performance on the neuropsychological tests unrelated to the training. These results are similar to those obtained in the current study. Altogether these findings should prompt the development of cognitive intervention programs that will allow direct amelioration in the real life activities of AD patients. The proposed intervention required few sessions to acquire and retain new learning, which have clinical application in the context of AD rehabilitation. To our knowledge, only a very limited number of published studies have already reported such an improvement in learning abilities following a 4-week intervention. Finally, the present study has the particularity that it required the caregiver's involvement for the training phase. The good understanding and participation of the caregivers in this study indicated that they can collaborate in the cognitive rehabilitation of their relatives suffering from AD. This is an important finding given the augmentation of cost restrictions in health institutions and management.

This study however presents some limitations. First of all, no improvement in quality of life was found. Such an improvement might have provided support to the fact that the intervention met functional needs. However, a lack of sensitivity of the tool used herein might explain the results. Although the multiple-baseline across subjects design is generally considered to be the most robust among the single case designs (Levine and Downey-Lamb 2005), it nevertheless has some limits. This design does not provide much evidence regarding the participant's characteristics that may interact with or moderate the effects of the intervention. In addition, it does not tell much about the generality of findings (Kazdin 2002). For example, the results might only be generalized to patients with caregivers showing particular personal features such as low burden, high motivation, and good learning skills. Another limitation is that all phases of this study were realized by the same individual (the first author of the study), allowing the introduction of an experimenter bias. However, all the assessments were conducted in a standardized manner, strictly following the administration and scoring instructions of the test manuals. In addition, this situation replicates the conditions of cognitive training in clinical settings where, most of the time, only one clinician will administer the training. The fact that Participant A was on citalopram for only 16 days when he was tested at baseline could potentially represent a threat to internal validity. Indeed, one might argue that the improvement on the task could be due to the positive effects of citalopram. However, the results of recent studies conducted in healthy adults receiving 10 to $40 \mathrm{mg}$ per day of citalopram (Rose et al 2006; Paul et al 2007), and in patients with mild-to-moderate 
$\mathrm{AD}$ receiving a mean dose of $35 \mathrm{mg}$ per day of citalopram together with a cholinesterase inhibitor (Caballero et al 2006) showed that citalopram had no impact on several measures of cognition at short (few weeks) and long-term (annual) time intervals. Therefore it is unlikely that the improvements registered by Participant A on the trained task were due to citalopram, especially given that his performance did not improve on the DRS-2. The amelioration on the trained task was measured using a different DMT for each participant. The DMTs were adapted from existing functional scales designed to specifically assess the amelioration of a forgotten IADL for each participant. Even though these instruments were adapted from relevant performance scales with good psychometric properties, we cannot presume that the modifications we introduced did not modify their validity and reliability. However, the fact that the caregiver's comments (in the practice journal) mirrored the results found on the 2 DMT suggests that these instruments had some validity. Despite these limitations, the inclusion of such measures allowed the training and the direct assessment of the performance of IADLs that were significant for the two participants. This is an original contribution with a strong clinical relevance.

In conclusion, this investigation supports the tolerability and preliminary efficacy of a new cognitive training program using EL and SR techniques designed to respond specifically to the needs of AD patients. However, these promising results will have to be replicated in future case studies or in a more robust research design such as a single/multiple subject design including a comparison with control tasks (Backman et al 1997). Nevertheless, interventions such as the one conducted in this study are needed in order to maintain or enhance $\mathrm{AD}$ patient's autonomy in daily living.

\section{Acknowledgments}

This study was supported by doctoral research grants awarded to Stéphanie Thivierge by Programme-réseau de Formation interdisciplinaire en recherche Santé et Vieillissement (FORMSAV), and by the Alzheimer Society of Canada in collaboration with the Fonds de la Recherche en Santé du Québec (FRSQ). Dr Martine Simard is supported by a 2005 NARSAD Young Investigator Award and is involved in research funded by the Alzheimer Society of Canada and the Canadian Institute of Health Research (CIHR), Institute of Aging.

\section{References}

Adam S, Van Der Linden M, Juillerat A-C, et al. 2000. The cognitive management of daily life activities in patients with mild to moderate Alzheimer's disease in a day care centre: A case report. Neuropsychol Rehabil, 10:485-509.
Ala TA, Berck LG, Popovich AM. 2005. Using the telephone to call for help and caregiver awareness in Alzheimer disease. Alzheimer Dis Assoc Disord, 19:79-84.

Backman CL, Harris SR, Chisholm JM, et al. 1997. Single-subject research in rehabilitation: a review of studies using $\mathrm{AB}$, withdrawal, multiple baseline, and alternating treatments designs. Arch Phys Med Rehabil, 78:145-53.

Baddeley A, Wilson BA. 1994. When implicit learning fails: amnesia and the problem of error elimination. Neuropsychologia, 32:53-68.

Barlow DH, Hersen M. 1984. Multiple baseline designs. Single case experimental designs: Strategies for studying behavior change. New York: Pergamon Press, pp. 209-51.

Baum C, Edwards DF. 1993. Cognitive performance in senile dementia of the Alzheimer's type: the Kitchen Task Assessment. Am J Occup Ther, 47:431-6.

Bier N, Desrosiers J, Gagnon L. 2006. Cognitive training interventions for normal aging, mild cognitive impairment and Alzheimer's. Can J Occup Ther, 73:26-35.

Bier N, Provencher V, Gagnon L, et al. 2008. New learning in dementia: Transfer and spontaneous use of learning in everyday life functioning. Two cases studies. Neuropsychol Rehabil, 18:204-35.

Bird M, Alexopoulos P, Adamowicz J. 1995. Success and failure in five case studies: Use of cued recall to ameliorate behaviour problems in senile dementia. Int J Geriatr Psychiatry, 10:305-11.

Birks J, Grimley Evans J, Iakovidou V, et al. 2000. Rivastigmine for Alzheimer's disease. Cochrane Database Syst Rev, 2:CD001191.

Birks J, Harvey RJ. 2006. Donepezil for dementia due to Alzheimer's disease. Cochrane Database Syst Rev, 4:CD001190.

Blessed G, Tomlinson BE, Roth M. 1968. Association between quantitative measures of dementing and senile change in the cerebral grey matter of elderly subjects. Br J Psychiatry, 114:797-811.

Bradshaw J, Saling M, Hopwood M, et al. 2004. Fluctuating cognition in dementia with Lewy bodies and Alzheimer disease is qualitatively distinct. J Neurol Neurosurg Psychiatry, 75:382-7.

Bravo G, Hébert R. 1997. Age and education specific reference values for the Mini-Mental and Modified Mini-Mental State Examinations derived from a non-demented elderly population. Int J Geriatr Psychiatry, 12:1008-18.

Brod M, Stewart AL, Sands L, et al. 1999. Conceptualization and measurement of quality of life in dementia: The dementia quality of life instrument (DQoL). Gerontologist, 39:25.

Caballero J, Hitchcock M, Beversdorf D, et al. 2006. Long-term effects of antidepressants on cognition in patients with Alzheimer's disease. J Clin Pharm Ther, 31:593-8.

Camp CJ, Foss JW, O'Hanlon AM, et al. 1996. Memory interventions for persons with dementia. Applied Cognitive Psychology, 10:193-210.

Canadian Study of Health and Aging. 1994. Canadian study of health and aging: study methods and prevalence of dementia. Can Med Assoc J, 150:899-913.

Canadian Study of Health and Aging. 1996. Consortium of Montreal and McGill Universities: Canadian Study of Health and Aging II-Manual of administration. Unpublished document.

Carney N, Chesnut RM, Maynard H, et al. 1999. Effect of cognitive rehabilitation on outcomes for persons with traumatic brain injury: A systematic review. J Head Trauma Rehabil, 14:277-307.

Cicerone KD, Dahlberg C, Kalmar K, et al. 2000. Evidence-based cognitive rehabilitation: recommendations for clinical practice. Arch Phys Med Rehabil, 81:1596-615.

Clare L, Wilson BA, Carter G, et al. 2001. Long-term maintenance of treatment gains following a cognitive rehabilitation intervention in early dementia of Alzheimer type: A single case study. Neuropsychol Rehabil, 11:477-94.

Cotrell V, Wild K, Bader T. 2006. Medication management and adherence among cognitively impaired older adults. J Gerontol Soc Work, 47:31-46.

Culberston WC, Zillmer EA. 2000. Tower of London. Toronto: Multi-Health Systems. 
Cummings JL, Mega M, Gray K, et al. 1994. The Neuropsychiatric Inventory: comprehensive assessment of psychopathology in dementia. Neurology, 44:2308-14.

Davis RN, Massman PJ, Doody RS. 2001. Cognitive intervention in Alzheimer disease: a randomized placebo-controlled study. Alzheimer Dis Assoc Disord, 15:1-9.

Delis DC, Kaplan EF, Kramer JH. 2000. Delis Kaplan Function System. San Antonio: Psychological Corporation.

Delis DC, Kramer JH, Kaplan EF, et al. 2000. The California Verbal Learning Test. Second Edition. New York: Psychological Corporation.

Evans JG, Wilcock G, Birks J. 2004. Evidence-based pharmacotherapy of Alzheimer's disease. Int J Neuropsychopharmacol, 7:351-69.

Folstein MF, Folstein SE, McHugh PR. 1975. "Mini-mental state”. A practical method for grading the cognitive state of patients for the clinician. $J$ Psychiatr Res, 12:189-98.

Gélinas I, Auer S, Gauthier S. 1996. Functional autonomy. Clinical diagnosis and management of Alzheimer's disease. London: Martin Dunitz, pp. 191-202.

Gélinas I, Gauthier L. 1994. [Évaluation de l'incapacité fonctionnelle dans la démence: Guide de l'utilisateur.] unpublished document.

Grandmaison E, Simard M. 2003. A critical review of memory stimulation programs in Alzheimer's disease. J Neuropsychiatry Clin Neurosci, 15:130-44.

Hebert LE, Scherr PA, Bienas JL, et al. 2003. Alzheimer disease in US population. Prevalence estimates using the 2000 census. Arch Neurol, 60:1119-22.

Hugues CP, Berg L, Danziger WL, et al. 1982. A new clinical scale for the staging of dementia. Br J Psychiatry, 140:566-72.

Jean L, Simard M, Van Reekum R, et al. 2007. Towards a cognitive stimulation program using an errorless learning paradigm in amnestic mild cognitive impairment: A case report study. Neuropsychiatr Dis Treat, 3:975-85.

Jurica PJ, Leitten CL, Mattis S. 2001. Dementia Rating Scale-2: Professional Manual. Lutz, Florida: Psychological Assessment Resources, Inc.

Kaplan EF, Goodglass H, Weintraub S. 1983. The Boston Naming Test. Boston: Kaplan and Goodglass.

Kazdin AE. 1982. Multiple-Baseline Designs. Single-Case Research Designs. New York: Oxford University Press, pp. 126-51.

Kazdin AE. 2002. Research design in clinical psychology. Fourth ed. Boston: Allyn and Bacon.

Lekeu F, Chicherio C, Van der Linden M, et al. 2000. Prise en charge des difficultés de mémoire prospective dans la maladie d'Alzheimer [Prospective memory rehabilitation in Alzheimer's disease]. Alzheimer, 3:17-20.

Lekeu F, Wojtasik V, Van der Linden M, et al. 2002. Training early Alzheimer patients to use a mobile phone. Acta Neurol Belg, 102:114-21.

Levine B, Downey-Lamb MM. 2005. Design and evaluation of rehabilitation experiments. In: Eslinger P (ed). Neuropsychological interventions: clinical research and practice. New York: The Guilford Press, pp. 80-102

Lezak MD. 2004. Neuropsychological Assessment. New York: Oxford University Press.

Loewenstein DA, Acevedo A, Czaja SJ, et al. 2004. Cognitive rehabilitation of mildly impaired Alzheimer disease patients on cholinesterase inhibitors. Am J Geriatr Psychiatry, 12:395-402.

Loewenstein DA, Amigo E, Duara R, et al. 1989. A new scale for the assessment of functional status in Alzheimer's disease and related disorders. $J$ Gerontol, 44:114-21.

Loewenstein DA, Duara R, Rubert MP, et al. 1995. Deterioration of functional capacities in Alzheimer's disease after a 1-year period. Int Psychogeriatr, 7:495-503.

Loy C, Schneider L. 2006. Galantamine for Alzheimer's disease and mild cognitive impairment. Cochrane Database Syst Rev, 1:CD001747.

Marson DC, Sawrie SM, Snyder S, et al. 2000. Assessing financial capacity in patients with Alzheimer disease: A conceptual model and prototype instrument. Arch Neurol, 57:877-84.
Mattis S. 2001. Dementia Rating Scale-2. Lutz, Florida: Psychological Assessment Ressources Inc.

McKhann G, Drachman D, Folstein M, et al. 1984. Clinical diagnosis of Alzheimer's disease: report of the NINCDS-ADRDA Work Group under the auspices of Department of Health and Human Services Task Force on Alzheimer's Disease. Neurology, 34:939-44.

Metzler-Baddeley C, Snowden JS. 2005. Brief report: errorless versus errorful learning as a memory rehabilitation approach in Alzheimer's Disease. J Clin Exp Neuropsychol, 27:1070-9.

Monsch AU, Mondi MW, Salmon DP, et al. 1995. Clinical Validity of the Mattis Dementia Rating Scale in Detecting Dementia of the Alzheimer Type. Arch Neurol, 52:899-904.

Moore DJ, Palmer BW, Patterson TL, et al. 2007. A review of performancebased measures of functional living skills. J Psychiatr Res, 41:97-118.

Parker RI, Hagan-Burke S. 2007. Useful effect size interpretations for single case research. Behav Ther, 38:95-105.

Paul MA, Gray GW, Love RJ, et al. 2007. SSRI effects on psychomotor performance: assessment of citalopram and escitalopram on normal subjects. Aviat Space Environ Med, 78:693-7.

Petersen RC. 2004. Mild cognitive impairment as a diagnostic entity. J Intern Med, 256:183-94.

Qiu C, De Ronchi D, Fratiglioni L. 2007. The epidemiology of the dementias: an update. Curr Opin Psychiatry, 20:380-5.

Rouleau I, Salmon DP, Butters N, et al. 1992. Quantitative and qualitative analyses of clock drawings in Alzheimer's and Huntington's disease. Brain Cogn, 18:70-87.

Rose EJ, Simonotto E, Spencer EP, et al. 2006. The effects of escitalopram on working memory and brain activity in healthy adults during performance of the n-back task. Psychophramacology (Berl), 185:339-47.

Salmon DP, Thal LJ, Butters N, et al. 1990. Longitudinal evaluation of dementia of the Alzheimer type: a comparison of 3 standardized mental status examinations. Neurology, 40:1225-30.

Schölzel-Dorenbos CJM, Ettema TP, Bos J, et al. 2007. Evaluating the outcome of intervention on quality of life in dementia: Selection of the appropriate scale. Int J Geriatr Psychiatry, 22:511-19.

Shay KA, Duke LW, Conboy T, et al. 1991. The clinical validity of the Mattis Dementia Rating Scale in staging Alzheimer's dementia. J Geriatr Psychiatry Neurol, 4:18-25.

Simard M, Sampson EL. 2008. Dementia: pharmacological and nonpharmacological treatments and guideline review. In: Tyrer P, Silk KR (eds). The Cambridge Textbook of Effective Treatments in Psychiatry. Cambridge: Cambridge University Press, pp. 217-43.

Sitzer DI, Twamley EW, Jeste DV. 2006. Cognitive training in Alzheimer's disease: a meta-analysis of the literature. Acta Psychiatr Scand, 114:75-90.

Skurla E, Rogers JC, Sunderland T. 1988. Direct assessment of activities of daily living in Alzheimer's disease. A controlled study. J Am Geriatr Soc, 36:97-103.

Stuss DT, Meiran N, Guzmen A, et al. 1996. Do long tests yield a more accurate diagnosis of dementia than short tests? A comparison of five neuropsychological tests. Arch Neurol, 53:1033-9.

Tuokko H, Hadjistavropoulos T, Miller JA, et al. 1992. The Clock Test: a sensitive measure to differentiate normal elderly from those with Alzheimer disease. J Am Geriatr Soc, 40:579-84.

Tyson PJ, Laws KR, Roberts KH, et al. 2005. A longitudinal analysis of memory in patients with schizophrenia. J Clin Exp Neuropsychol, 27:718-34.

Wilson B, Cockburn J, Baddeley A, et al. 1989. The development and validation of a test battery for detecting and monitoring everyday memory problems. J Clin Exp Neuropsychol, 11:855-70.

Zanetti O, Zanieri G, Di Giovanni G, et al. 2001. Effectiveness of procedural memory stimulation in mild Alzheimer's disease patients: A controlled study. Neuropsychol Rehabil, 11:263-72. 